\title{
Densitometría ósea en mujeres chilenas normales en edad fértil: correlación con valores referenciales y variables antropométricas
}

\author{
G abriel Lobo $\mathbf{S}^{1,2}$, D avid Ladrón de G uevara $\mathrm{H}^{2}$, \\ Alexandra Zerboni $\mathbf{A}^{\text {la }}$. \\ Dual X-ray absorptiometry [DEXA] in \\ normal Chilean women. Correlation \\ between reference values and \\ anthropometric variables
} \begin{abstract}
ber of standard deviations from reference values in similar populations. However, these reference values have not been obtained locally and there may be discrepancies between Chilean and other Hispanic populations. Aim: To evaluate the results of DEXA in healthy Chilean women and to correlate them with reference values and anthropometric parameters. Material and Method: We studied prospectively 166 normal women aged between 15 and 55 years, using a Hologic QDR 1000 equipment, yielding bone mineral density (BMD), bone mineral content (BMC) and bone area (A) in the lumbar spine (LS) and femoral neck (FN). These bone densitometric variables were correlated with weight, height and age of women and compared against the reference values of the Hologic equipment. Relation between BMC and A was evaluated using a regression analysis. Results: BMD and BMC were lower to reference values in most age groups, especially for LS. There was a significant correlation of BMD with A in LS (r: 0.278, $p \varangle 0.001$ ), weight in both LS (r: 0.382, p $\varangle 0.0001$ ) and FN (r: 0.266, p $\varangle 0.001$ ), height in LS ( $\mathrm{r}: 0.258, \mathrm{p} \varangle 0.001$ ) and age in FN ( $\mathrm{r}$ : $-0.231, \mathrm{p} \varangle 0.01)$. Only in LS, regression analysis between A and BMC did not show direct proportionality. Conclusions: BMD of healthy Chilean women was lower than the reference values of the Hologic equipment for most age groups. Bone densitometric variables are non uniformly influenced by weight, height and size of bone structures (A) in normal women, especially in LS. Correct interpretation of DEXA requires the validation of reference values and to assess the value of bone area measurements (Rev Méd Chile 2004; 132: 681-90).
\end{abstract}

Background: Bone mineral density results are expressed as the num-

(Key Words: Absortiometry, photon; Bone mineral contact; Bone mineral density; Densitometry)

Recibido el 16 de enero, 2003. Aceptado en versión corregida el 7 de abril, 2004.

Resultados parciales de un proyecto cooperativo internacional ( $\left.n^{\circ} 8190 / R B\right)$ con financiamiento de OIEA (11 países de Europa, Asia y América).

${ }^{1}$ Servicio de Medicina Nuclear y de Imagenología Clínica Indisa, Universidad Andrés Bello.

${ }^{2}$ Unidad de Medicina Nuclear, Hospital San Juan de Dios. División Ciencias Médicas Occidente, Facultad de Medicina, Universidad de Chile.

aTecnóloga Médica

Correspondencia a: Gabriel Lobo S. Unidad de Medicina Nuclear, CDT Hospital San Juan de Dios. Portales 3239, Quinta Normal, Santiago, Chile. Fono: 4506869. FonoFax: 6825314. E-mail: globo@machi.med.uchile.cl 
$\mathrm{L}^{\mathrm{a}}$ a densitometría ósea bifotónica con rayos $\mathrm{X}$ es considerada el método no invasivo de referencia para medir la masa mineral ósea ${ }^{1}$. Si bien la tecnología utilizada ha alcanzado un elevado nivel de confiabilidad, precisión y reproducibilidad, su interpretación debe ser optimizada, para asegurar que las variaciones observadas reflejen el contenido mineral óseo y no otras variables asociadas, de origen técnico o biológico.

Los resultados de la densitometría ósea se expresan a través de 3 variables osteodensitométricas, el contenido mineral óseo (CMO), el área ósea (A) y la densidad mineral ósea (DMO). Se entiende por CMO la cantidad total de mineral expresada en gramos en un segmento óseo determinado. El A corresponde a una superficie en $\mathrm{cm} 2$, que depende del tamaño del hueso y es determinada de forma automática en base a algoritmos matemáticos que consideran gradientes de densidad para definir los límites de la estructura ósea evaluada. La DMO es el CMO corregido por el A, se expresa en $\mathrm{g} / \mathrm{cm}^{2}$ y es utilizada como medio de estandarización para disminuir el efecto de diferencias en el tamaño óseo entre individuos y facilitar la comparación de un individuo con una población de referencia ${ }^{2}$.

Estas variables se interrelacionan entre sí y su comportamiento puede diferir en distintas situaciones de manera que no parece ser equivalente, por ejemplo, utilizar indistintamente el CMO o la DMO para determinar el riesgo de fractura, como ha sido demostrado en estudios recientes ${ }^{3}$.

Entre los principales factores conocidos que pueden afectar dichas variables osteodensitométricas se encuentran aquellos dependientes de la técnica, cuya influencia puede minimizarse a través de una estandarización y control de calidad adecuados $^{4,5}$ y otras condiciones, cuyo efecto es más controvertido, relacionadas con características propias de los individuos, especialmente parámetros antropométricos y asociadas a ellas ${ }^{2}$. Así se ha discutido la posible influencia del tamaño y profundidad de las estructuras óseas, grosor y características de los tejidos blandos, entre otros, que serían factores que pueden modificar las medidas efectuadas, sin reflejar el verdadero contenido mineral óseo.

Estas condiciones pueden tener especial relevancia, por cuanto la interpretación de los resulta- dos densitométricos depende de comparaciones con mediciones efectuadas en poblaciones referenciales normativas incorporadas en los equipos, muchas de cuyas características se ignoran y que pueden ser distintas a las de la población local, independientemente de consideraciones de muestreo estadístico, étnicas o genéticas. Esta situación, frecuentemente no considerada en diversos trabajos clínicos y epidemiológicos nacionales e internacionales tratantes del tema ${ }^{2,6-8}$, puede generar errores, tanto en la definición de conceptos básicos como osteopenia y osteoporosis, como en la interpretación de resultados, ya sea individuales 0 en estudios poblacionales ${ }^{2,4}$.

El objetivo de este trabajo es conocer algunas características de los resultados de la densitometría ósea en población de mujeres chilenas normales y su correlación con valores de referencia y parámetros antropométricos.

\section{Pacientes y MÉtodos}

Se estudiaron 166 mujeres con edades entre 15 y 50 años, pertenecientes a la población urbana de Santiago de Chile, sin evidencias de enfermedad, que cumplían con los criterios de exclusión que se señalan en la Tabla 1, cuyo objetivo era evitar la inclusión en la serie de mujeres a estudiar, de aquellas que pudieran tener factores condicionantes o causales de cambios transitorios o permanentes de su contenido mineral óseo. Estos criterios fueron definidos de acuerdo a instructivos del Organismo Internacional de Energía Ató-

\section{Tabla 1. Criterios de exclusión}

1. Escoliosis moderada a severa.

2. Enfermedad crónica conocida, pasada 0 presente, con más de 3 meses de duración.

3. Uso crónico (>1 mes) de cualquier medicación, excepto suplementos vitamínicos en la dieta.

4. Fracturas previas sin clara justificación traumática.

5. Inmovilización prolongada ( $>1$ mes).

6. Exposición a metales tóxicos o irradiación.

7. Trastornos menstruales. 
mica para estudios osteodensitométricos poblacionales en normales (Comparative International Studies of Osteoporosis using isotope related techniques. NAHRES-31 IAEA Vienna 1966 1-8) y que consideran la exclusión de portadores de enfermedades crónicas en general, exposición a condiciones que pueden afectar la densidad ósea (inmobilización, medicamentos y otras substancias), sintomatología que pueda indicar algún trastorno de importancia aunque no conocido por el sujeto (trastornos menstruales) o que hagan sospechar una fragilidad ósea anormal. Estas personas se reclutaron entre las funcionarias de dos centros asistenciales de Santiago, uno del sector público y otro del privado. A todas ellas se les efectuó una encuesta destinada a evaluar su estado de normalidad clínica y nutricional.

Las participantes fueron estudiadas mediante un densitómetro HOLOGIC QDR 1000, sometido a los controles de calidad periódicos propios de dicho equipo y a un control internacional, a través del Phantom Europeo de control de calidad (European Spine Phantom: ESP), cuyo resultado reveló una precisión y exactitud satisfactorias en las mediciones (Tabla 2), especialmente en el rango de densidad mineral ósea cercana a $1 \mathrm{~g} /$ $\mathrm{cm}^{2}$, que corresponde al encontrado en la población en estudio para columna vertebral y cuello femoral.

Se establecieron los valores observados de las variables osteodensitométricas: DMO, CMO y A y se calcularon promedios, desviaciones estándar y Z scores para el grupo total y según grupos etarios de 5 años. Se realizó un análisis de correlación entre las variables osteodensitométricas con edad y parámetros relacionados con el tamaño corporal, así como el análisis comparativo entre los valores observados y los valores de referencia incorporados al densitómetro (base de datos Hologic 1990 en 1.500 individuos), actualizada con posterioridad a nuestro trabajo por la firma Hologic según el estudio multicéntrico «Third National Health and Nutrition Examination Survey» (NHANES III). Para esta última comparación fue necesario segmentar la muestra en rangos etarios similares a los de la base de datos Hologic (Tabla 6), que no es posible reagrupar.

La relación entre el CMO y el A se analizó tanto para columna vertebral como para cuello femoral según procedimiento de Prentice et $\mathrm{al}^{2}$. En dicho modelo estadístico, la variable dependiente es el logaritmo natural de CMO y la variable independiente es el logaritmo natural del área del hueso. En este análisis se postula un coeficiente de regresión igual a 1, lo que significa que existiría una relación directa simple entre el CMO y el A, y que el área permite ajustar correctamente el CMO según el tamaño de las estructuras óseas y expresarlo como DMO. Si dicho coeficiente es distinto de 1, esta última condición no se cumplinía, significando que la DMO podría no ser un índice adecuado, por cuanto estaría afectado por variables dependientes del tamaño corporal. Los parámetros antropométricos de la población estudiada agrupada según rango etario, se presentan en la Tabla 3.

Para el análisis estadístico comparativo de los datos obtenidos en la población normal estudiada, respecto a la base de datos incorporada en el equipo Hologic, se efectuó una comparación

\section{Tabla 2. Variables osteodensitométricas obtenidas con el European Spine Phantom en el densitómetro H ologic Q D R 1000, determinadas en 10 mediciones consecutivas (promedio \pm SD )}

\begin{tabular}{|lcccc|}
\hline DMO $\left(\mathrm{g} / \mathrm{cm}^{2}\right)$ & DMO Standard $\left(\mathrm{g} / \mathrm{cm}^{2}\right)$ & CMO $(\mathrm{g})$ & A $\left(\mathrm{cm}^{2}\right)$ & DMO referencia $\left(\mathrm{g} / \mathrm{cm}^{2}\right)$ \\
\hline $0,490 \pm 0,019$ & $0,526 \pm 0,02$ & $4,486 \pm 0,169$ & $9,152 \pm 0,108$ & 0,5 \\
$0,932 \pm 0,00089$ & $1,002 \pm 0,00095$ & $9,902 \pm 0,129$ & $10,628 \pm 0,182$ & 1 \\
$1,359 \pm 0,0021$ & $1,462 \pm 0,0023$ & $15,049 \pm 0,217$ & $11,074 \pm 0,238$ & 1,5 \\
\hline
\end{tabular}

DMO: densidad mineral ósea; DMO Standard: DMO corregida de acuerdo al error sistemático de los densitómetros de distintas marcas*; CMO: contenido mineral óseo; A: área ósea evaluada. *Para los equipos Hologic se aplica la fórmula $\mathrm{BMD}=1000\left[\mathrm{BMD}_{\text {Hologic }} \times 1,0755\right]$. 
Tabla 3. Parámetros antropométricos en mujeres chilenas normales

\begin{tabular}{|lcccc|}
\hline Rango etario & $\mathrm{n}$ & $\begin{array}{c}\text { Peso }(\mathrm{kg}) \\
(\xi \pm \mathrm{SD})\end{array}$ & $\begin{array}{c}\text { Talla }(\mathrm{cm}) \\
(\xi \pm \mathrm{SD})\end{array}$ & $\begin{array}{c}\text { Indice de masa corporal } \\
(\xi \pm \mathrm{SD})\end{array}$ \\
\hline $16-20$ & 12 & $58,38( \pm 21,3)$ & $155,41( \pm 5,36)$ & $24,10( \pm 8,12)$ \\
$21-25$ & 18 & $57,17( \pm 9,25)$ & $160,36( \pm 5,50)$ & $22,27( \pm 3,82)$ \\
$26-30$ & 26 & $57,91( \pm 5,91)$ & $157,34( \pm 4,24)$ & $23,37( \pm 2,00)$ \\
$31-35$ & 26 & $62,00( \pm 9,55)$ & $159,26( \pm 5,79)$ & $24,44( \pm 3,52)$ \\
$36-40$ & 32 & $61,07( \pm 8,82)$ & $157,15( \pm 6,66)$ & $24,68( \pm 2,85)$ \\
$41-45$ & 24 & $62,67( \pm 14,84)$ & $157,00( \pm 6,66)$ & $25,07( \pm 4,78)$ \\
$46-50$ & 28 & $59,88( \pm 14,18)$ & $157,75( \pm 5,60)$ & $24,09( \pm 5,09)$ \\
Total & 166 & $60,13( \pm 11,84)$ & $157,90( \pm 5,83)$ & $24,09( \pm 4,41)$ \\
\hline
\end{tabular}

$\xi=$ promedio.

Tabla 4. Variables osteodensitométricas obtenidas en columna vertebral lumbar de mujeres chilenas normales (L1-L4)

\begin{tabular}{|lcccc|}
\hline Rango etario & $\mathrm{n}$ & $\begin{array}{c}\text { Densidad mineral ósea } \\
(\xi \pm \mathrm{SD})\left(\mathrm{g} / \mathrm{cm}^{2}\right)\end{array}$ & $\begin{array}{c}\text { Contenido mineral óseo } \\
(\xi \pm \mathrm{SD})(\mathrm{g})\end{array}$ & $\begin{array}{c}\text { Área } \\
(\xi \pm \mathrm{SD})(\mathrm{cm} 2)\end{array}$ \\
\hline $16-20$ & 12 & $0,964( \pm 0,08)$ & $50,85( \pm 7,92)$ & $52,57( \pm 5,01)$ \\
$21-25$ & 18 & $1,003( \pm 0,12)$ & $54,02( \pm 9,81)$ & $53,62( \pm 5,27)$ \\
$26-30$ & 26 & $0,980( \pm 0,07)$ & $53,06( \pm 6,39)$ & $54,17( \pm 3,77)$ \\
$31-35$ & 26 & $0,995( \pm 0,08)$ & $54,68( \pm 6,69)$ & $54,85( \pm 3,65)$ \\
$36-40$ & 32 & $0,989( \pm 0,13)$ & $55,15( \pm 9,82)$ & $55,50( \pm 5,15)$ \\
$41-45$ & 24 & $0,994( \pm 0,14)$ & $55,09( \pm 10,48)$ & $55,22( \pm 4,89)$ \\
$46-50$ & 28 & $0,956( \pm 0,12)$ & $54,31( \pm 10,02)$ & $56,55( \pm 5,14)$ \\
Total & 166 & $0,983( \pm 0,113)$ & $54,16( \pm 8,85)$ & $54,92( \pm 4,75)$ \\
\hline
\end{tabular}

$\xi=$ promedio.

múltiple con corrección de Bonferroni (Tablas 6 y 7), y para el estudio de correlación entre parámetros antropométricos y variables osteodensitométricas se utilizaron pruebas de regresión lineal (Tabla 8). Para evaluar significación en el análisis de Prentice se realizó prueba t de student con $n$ 2 grados de libertad. El análisis descriptivo general e inferencial de los datos se realizó mediante el software estadístico STATA 5.0. Se consideró diferencias significativas valores de $\mathrm{p}<0,05$.

\section{RESULTADOS}

Los resultados de las variables osteodensitométricas en la columna vertebral y cuello femoral se presentan en las Tablas 4 y 5, observándose cifras comparables en los distintos grupos etarios.

Los resultados de la comparación entre los valores de DMO observados en la población normal estudiada y los de referencia incorporados en el equipo Hologic para columna vertebral y cuello femoral se muestran en la Tabla 6, Figuras 1 y 2. Se aprecia una tendencia de los valores observados a ser más bajos que los valores de referencia, obteniéndose diferencias significativas $(p<0,05)$ en la mayoría de los grupos etarios en cuello femoral y para los grupos 28-32, 33-37 y 3842 años en columna vertebral ( $p<0,05)$.

Los valores y distribución del Z score positivo o negativo, para columna vertebral y cuello femoral se muestran en la Tabla 7. Se aprecia un 
Tabla 5. Variables osteodensitométricas obtenidas en cuellos femorales de mujeres chilenas normales

\begin{tabular}{|ccccc|}
\hline Rango etario & $\mathrm{n}$ & $\begin{array}{c}\text { Densidad mineral ósea } \\
(\xi \pm \mathrm{SD})\left(\mathrm{g} / \mathrm{cm}^{2}\right)\end{array}$ & $\begin{array}{c}\text { Contenido mineral óseo } \\
(\xi \pm \mathrm{SD})(\mathrm{g})\end{array}$ & $\begin{array}{c}\text { área } \\
(\xi \pm \mathrm{SD})\left(\mathrm{cm}^{2}\right)\end{array}$ \\
\hline $16-20$ & 12 & $0,835( \pm 0,09)$ & $3,89( \pm 0,47)$ & $4,73( \pm 0,25)$ \\
$21-25$ & 18 & $0,864( \pm 0,10)$ & $4,24( \pm 0,62)$ & $4,88( \pm 0,31)$ \\
$26-30$ & 26 & $0,815( \pm 0,10)$ & $3,97( \pm 0,50)$ & $4,92( \pm 0,30)$ \\
$31-35$ & 26 & $0,813( \pm 0,07)$ & $3,99( \pm 0,46)$ & $4,89( \pm 0,34)$ \\
$36-40$ & 32 & $0,811( \pm 0,10)$ & $4,04( \pm 0,57)$ & $5,02( \pm 0,44)$ \\
$41-45$ & 24 & $0,779( \pm 0,08)$ & $3,89( \pm 0,54)$ & $5,00( \pm 0,35)$ \\
$46-50$ & 28 & $0,781( \pm 0,11)$ & $3,90( \pm 0,53)$ & $5,03( \pm 0,35)$ \\
Total & 166 & $0,811( \pm 0,10)$ & $3,89( \pm 0,47)$ & $4,95( \pm 0,35)$ \\
\hline
\end{tabular}

$\xi=$ promedio.

\section{Tabla 6. Comparación de los valores de referencia H ologic de D M O con los obtenidos en mujeres chilenas normales}

\begin{tabular}{|cccccccc|}
\hline $\begin{array}{l}\text { Rango } \\
\text { etario }\end{array}$ & $\mathrm{n}$ & $\begin{array}{c}\text { Hologic (VR) } \\
\text { DMO }(\xi \pm \text { SD) } \\
\left(\mathrm{g} / \mathrm{cm}^{2}\right)\end{array}$ & $\begin{array}{c}\text { Columna vertebral } \\
\text { VO } \\
\text { DMO }(\xi \pm \text { SD) } \\
\left(\mathrm{g} / \mathrm{cm}^{2}\right)\end{array}$ & $\mathrm{p}$ & $\begin{array}{c}\text { Hologic (VR) } \\
\text { DMO }(\xi \pm S D) \\
\left(\mathrm{g} / \mathrm{cm}^{2}\right)\end{array}$ & $\begin{array}{c}\text { Cuello femoral } \\
\text { VOO }\end{array}$ & $\mathrm{p}$ \\
$\left(\mathrm{g} / \mathrm{cm}^{2}\right)$ & \\
\hline $19-22$ & 13 & $1,019 \pm 0,11$ & $0,972 \pm 0,099$ & NS & $0,895 \pm 0,1$ & $0,843 \pm 0,083$ & $<0,05$ \\
$23-27$ & 23 & $1,040 \pm 0,11$ & $1,007 \pm 0,106$ & NS & $0,894 \pm 0,1$ & $0,865 \pm 0,110$ & NS \\
$28-32$ & 24 & $1,047 \pm 0,11$ & $0,977 \pm 0,068$ & $<0,005$ & $0,886 \pm 0,1$ & $0,793 \pm 0,084$ & $<0,05$ \\
$33-37$ & 29 & $1,041 \pm 0,11$ & $0,999 \pm 0,109$ & $<0,05$ & $0,871 \pm 0,1$ & $0,835 \pm 0,089$ & $<0,05$ \\
$38-42$ & 31 & $1,024 \pm 0,11$ & $0,972 \pm 0,131$ & $<0,05$ & $0,850 \pm 0,1$ & $0,773 \pm 0,096$ & $<0,05$ \\
$43-47$ & 21 & $0,999 \pm 0,11$ & $0,989 \pm 0,146$ & NS & $0,826 \pm 0,1$ & $0,782 \pm 0,102$ & NS \\
$48-52$ & 19 & $0,967 \pm 0,11$ & $0,960 \pm 0,132$ & NS & $0,797 \pm 0,1$ & $0,795 \pm 0,111$ & NS \\
\hline
\end{tabular}

DMO: densidad mineral ósea; VO: valores observados; VR: valores de referencia; $\xi$ : promedio; NS: valor de $\mathrm{p}$ no significativo.

*Esta disposición de grupos etarios fue adoptada para realizar la comparación con la base de datos HOLOGIC. Los individuos menores de 19 años no fueron considerados en este análisis por su escaso número.

predominio global significativo de Z score negativos en ambas localizaciones ( $p<0,001$ ). Al analizar individualmente por grupos etarios, esta tendencia fue significativa para los intervalos 2630, 31-35 y 41-45 años en cuello femoral, y 26-30 años en columna vertebral $(p<0,05)$.

Las correlaciones entre variables osteodensitométricas y antropométricas se muestran en la Tabla 8. Se demostró correlación significativa entre la DMO de columna vertebral y cuello femoral (r: 0,6027, p <0,0001), de DMO con el peso en columna ( $\mathrm{r}: 0,3821, \mathrm{p}<0,0001$ ) y cuello femoral ( $\mathrm{r}: 0,2661, \mathrm{p}<0,001$ ), de DMO con la talla ( $\mathrm{r}: 0,2578, \mathrm{p}<0,001)$ y A (r: 0,2778, $\mathrm{p}<0,001$ ) sólo para columna, de DMO con edad sólo para cuello femoral ( $r$ : $-0,2309, p<0,01$ ). Las A de columna vertebral y cuello femoral se correlacionaron entre sí ( $r: 0,5227, p<0,0001)$ y con el peso ( $r: 0,2876$ en columna, $\mathrm{r}$ : 0,3716 en cuello femoral), talla ( $\mathrm{r}$ : 0,6239 en columna, r: 0,4555 en cuello femoral) y edad ( $r$ : 0,2478 en columna, r: 0,2794 en cuello femoral) ( $p<0,001)$. 
Tabla 7. Valores y distribución del parámetro «Z » establecido según base de datos $\mathrm{H}$ ologic para columna vertebral y cuello femoral en mujeres chilenas normales

\begin{tabular}{|c|c|c|c|c|c|c|c|}
\hline \multirow[b]{2}{*}{ Rango etario } & \multicolumn{4}{|c|}{ Columna vertebral } & \multicolumn{3}{|c|}{ Cuello femoral } \\
\hline & $\mathrm{n}$ & $\xi \pm \mathrm{SD}$ & $\% \mathrm{Z}(-)$ & $\mathrm{p}$ & $\xi \pm \mathrm{SD}$ & $\% \mathrm{Z}(-)$ & $\mathrm{p}$ \\
\hline $16-20$ & 12 & $-0,475 \pm 0,76$ & 67 & NS & $-0,111 \pm 0,73$ & 60 & NS \\
\hline $21-25$ & 18 & $-0,281 \pm 1,07$ & 67 & NS & $-0,297 \pm 1,07$ & 67 & NS \\
\hline $26-30$ & 26 & $-0,606 \pm 0,67$ & 85 & $<0,05$ & $-0,751 \pm 1,06$ & 72 & $<0,05$ \\
\hline $31-35$ & 26 & $-0,428 \pm 0,77$ & 73 & NS & $-0,548 \pm 0,75$ & 68 & $<0,05$ \\
\hline $36-40$ & 32 & $-0,292 \pm 1,25$ & 53 & NS & $-0,452 \pm 1,02$ & 72 & NS \\
\hline $41-45$ & 24 & $-0,162 \pm 1,35$ & 79 & NS & $-0,541 \pm 0,84$ & 79 & $<0,05$ \\
\hline $46-50$ & 28 & $-0,182 \pm 1,12$ & 57 & NS & $-0,289 \pm 1,16$ & 56 & NS \\
\hline Total & 166 & $-0,337 \pm 1,04$ & 66 & $<0,05$ & $-0,472 \pm 0,98$ & 69 & $<0,05$ \\
\hline
\end{tabular}

$\xi$ : promedio, NS: Valor de p no significativo.

Tabla 8. Correlación entre variables antropométricas y osteodensitométricas en mujeres chilenas normales

\begin{tabular}{|lccccccc|}
\hline & & Peso & Talla & Edad & A CF & A CV & DMO CF \\
\hline Á CF & $\mathrm{r}$ & 0,3716 & 0,4555 & 0,2794 & & & \\
& $\mathrm{p}$ & $<0,05$ & $<0,05$ & $<0,05$ & & & \\
Á CV & $\mathrm{r}$ & 0,2876 & 0,6239 & 0,2478 & 0,5227 & & \\
& $\mathrm{p}$ & $<0,05$ & $<0,05$ & $<0,05$ & $<0,05$ & & \\
DMO CF & $\mathrm{r}$ & 0,2661 & 0,1094 & $-0,2309$ & $-0,1046$ & 0,0936 & \\
& $\mathrm{p}$ & $<0,05$ & $\mathrm{NS}$ & $<0,05$ & NS & NS & \\
DMO CV & $\mathrm{r}$ & 0,3821 & 0,2578 & $-0,0260$ & 0,2692 & 0,2778 & 0,6027 \\
& $\mathrm{p}$ & $<0,05$ & $<0,05$ & NS & $<0,05$ & $<0,05$ & $<0,05$ \\
\hline
\end{tabular}

DMO: densidad mineral ósea; CMO: contenido mineral óseo; A: área ósea evaluada; CV: columna vertebral; CF: cuello femoral; r: coeficiente de correlación r; p: valor de p.

Al aplicar el modelo de regresión lineal propuesto por Prentice y cols, se encontró que en la columna vertebral el coeficiente de regresión obtenido entre los logaritmos naturales del CMO y del A fue 1,356 (error estándar: 0,099), que difiere significativamente de $1(\mathrm{p}<0,05)$. Esto indica que en dicha estructura las relaciones entre el CMO y el A no corresponden a una proporción directa simple, y parte de las variaciones de la DMO (CMO/A) en una población serán debidas a diferencias en el tamaño óseo entre los individuos. Esta condición no se daría en el caso de cuello femoral en nuestra casuística, por cuanto el coeficiente de regresión encontrado 0,810 (error estándar: 0,142), no fue significativamente diferente de 1.

\section{DISCUSIÓN}

La densitometría ósea bifotónica con rayos $\mathrm{X}$ constituye actualmente un aporte esencial en la evaluación de pacientes con sospecha de osteoporosis, en la estimación del riesgo de fractura y en el control de su tratamiento ${ }^{1,9,10}$. Si bien la técnica ha experimentado un desarrollo en cuanto a precisión, exactitud, acortamiento de los tiempos de examen, disminución de las dosis de radiación y estabilidad de las calibraciones ${ }^{2,11,12}$, persisten algunas interrogantes referentes principalmente a la interpretación del estudio. Esta última se basa principalmente en la comparación de los valores obtenidos respecto a cifras normativas de referencia, que permiten establecer el $\mathrm{Z}$ 


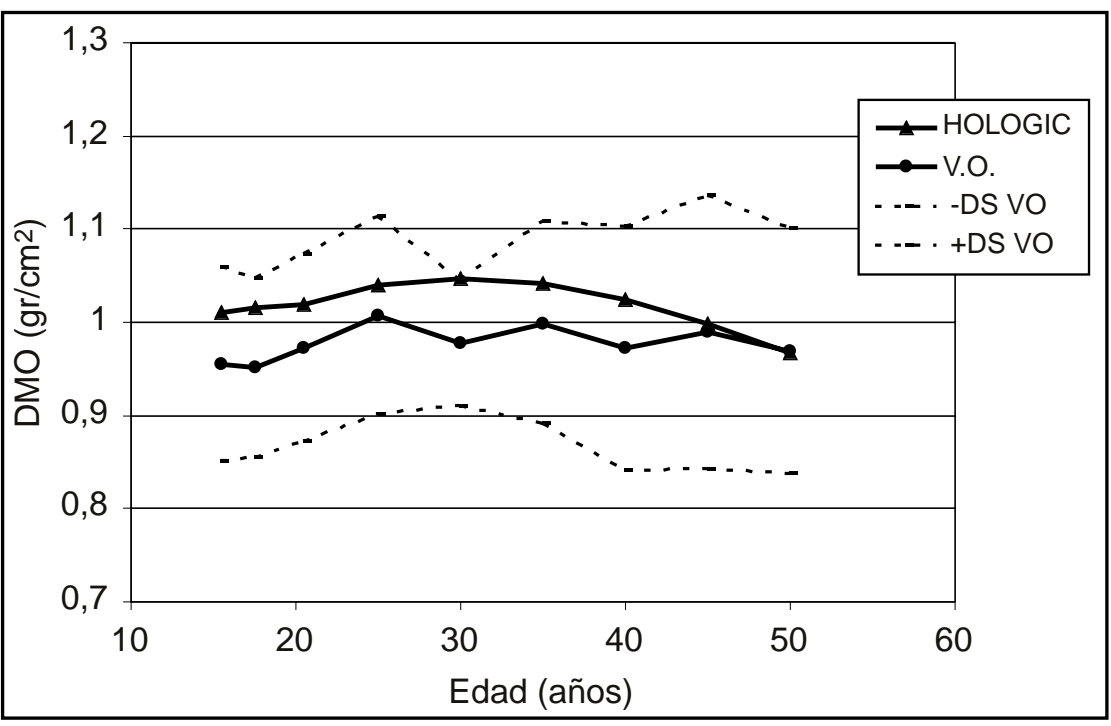

Figura 1. Valores de DMO de columna vertebral en mujeres chilenas normales y su comparación con valores de referencia Hologic.

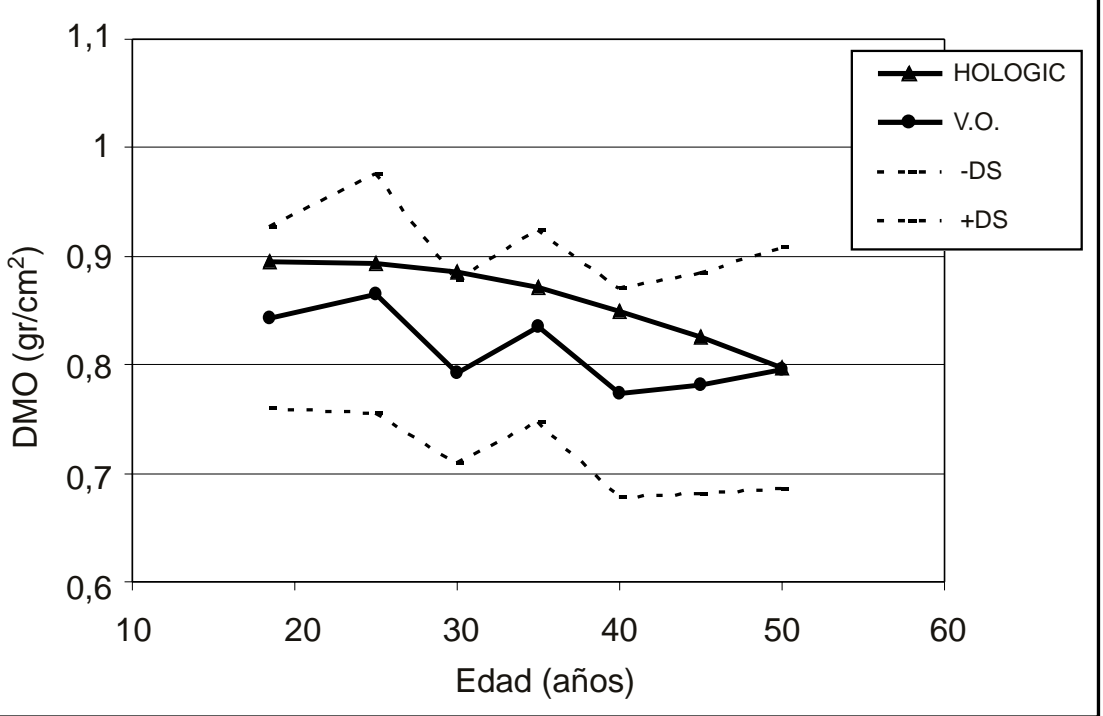

Figura 2. Valores de DMO de cuello femoral en mujeres chilenas normales y su comparación con valores de referencia Hologic.

score [DMO observada - DMO promedio para la edady/desviación estándar de la población] y el T score [DMO observada - DMO promedio de un adulto joven $/$ desviación estándar de un adulto joven], que a su vez son la base de los criterios clasificatorios de normalidad, osteopenia y osteoporosis, de acuerdo al criterio establecido por la $\mathrm{OMS}^{12}$.

En este estudio se describen los valores de las variables osteodensitométricas de una población de mujeres normales en edad fértil, se correlacionan con variables antropométricas y se comparan las cifras de densidad ósea con los valores de referencia provistos por el fabricante del densitómetro con el cual se efectuaron las mediciones.

La población normal estudiada presenta una relativa homogeneidad de los parámetros antropométricos y de las variables osteodensitométricas entre los grupos etarios evaluados. La comparación entre los valores de DMO observados y los referenciales, muestra diferencias en algunos de los grupos etarios, siendo en general más bajas las cifras de DMO en la población estudiada. Similar tendencia se aprecia al evaluar la distribución de 
los Z scores, evidenciándose un predominio de los valores negativos, lo que indica que la población normal estudiada tiene cifras de DMO predominantemente inferiores al promedio de la población referencial considerada normal por Hologic. $\mathrm{Si}$ las dos poblaciones fueran comparables, se esperaría encontrar una distribución relativamente similar entre la frecuencia de los Z scores positivos y negativos.

Se encontraron, además, correlaciones entre las variables osteodensitométricas y los parámetros antropométricos que indican, cómo ha sido demostrado en otros estudios ${ }^{2}$, un grado de dependencia entre estos factores. Todas estas condiciones pueden incidir en la correcta interpretación de los resultados del examen.

En efecto, existen problemas aún no resueltos en la construcción de las bases de datos de valores de referencia incorporados en los densitómetros $^{13}$ y utilizados en la interpretación de los estudios. Diversas investigaciones han demostrado la complejidad de las relaciones de las variables osteodensitométricas entre sí y con parámetros antropométricos ${ }^{2,11}$, así como la dificultad de corregir los efectos de dichos parámetros sobre las cifras de $\mathrm{DMO}^{2}$. Esta condición puede incidir en que determinadas bases de datos referenciales pudieran no ser aplicables en poblaciones en las cuales los parámetros antropométricos sean sustantivamente diferentes. Los resultados de este trabajo apoyan la validez de las interrogantes planteadas en este sentido, por cuanto como se señaló, se encontraron diferencias significativas en nuestra población normal respecto a la base de datos Hologic, especialmente en edades críticas para la definición del $\mathrm{T}$ score, $\mathrm{y}$ además se observó una predominancia de Z score negativos en los individuos normales estudiados.

Estas diferencias podrían deberse a la existencia de condiciones antropométricas distintas en la población de referencia con que se construyó la base de datos normativa, respecto a las mujeres chilenas normales estudiadas o a defectos de diseño en la construcción de dicha base de datos, como, por ejemplo, criterios de inclusión variables o sesgados y número insuficiente de individuos en algunos grupos etarios, que como ha sido demostrado, pueden introducir cambios muy significativos en los valores de los scores $\mathrm{T}$ y $\mathrm{Z}^{14}$. Por este motivo, se ha propuesto el uso de una base de datos común para los distintos densitómetros (NHANES III) ${ }^{14,15}$. La base de datos Hologic utilizada corresponde a aquella disponible previamente a la incorporación de los valores normativos NHANES III en los equipos Hologic y Lunar, actualmente en uso.

En éste y otros trabajos se demuestra que la relación entre los parámetros antropométricos y la DMO es diferente según la localización anatómica evaluada $^{2,11}$, de manera que la DMO de la columna vertebral se correlacionó con peso, talla y tamaño del hueso evaluado (A), en tanto que en el cuello femoral sólo se correlacionó con el peso. Estos hallazgos son, a su vez, concordantes con el análisis de regresión efectuado según el modelo de Prentice ${ }^{2}$ en que se demuestra una insuficiente corrección del CMO por el área ósea en la columna vertebral, debido a la carencia de proporcionalidad directa simple entre ambas variables.

Las interacciones entre DMO, CMO, A y parámetros dependientes del tamaño corporal parecen entonces ser complejas, de allí la dificultad para corregir su efecto, resultando deseable su ponderación cuando se construyen las bases de datos normativas, independientemente de consideraciones de tipo étnico o genético.

Los análisis efectuados en torno al área ósea son de especial interés. La carencia de proporcionalidad directa entre el CMO y el $\mathrm{A}^{2}$, corroborada en este estudio para la columna vertebral, indica que en la ecuación $\mathrm{CMO}=\mathrm{DMO} \times \mathrm{A}, \mathrm{DMO}$ no es una constante, por lo que modificaciones del área pueden no acompañarse de cambios proporcionales del CMO y resultar en cambios inexistentes en la DMO. Existen estudios prospectivos ${ }^{11}$ que demuestran que el A puede ser afectada por variaciones en la composición corporal, determinando cambios en la DMO sin que varíe el CMO. Por otra parte, como se señaló, la corrección por el tamaño óseo (a través del área) sobre el CMO es incompleta y por ello, los huesos de mayor tamaño tenderían a presentar valores más altos de DMO $\left(\mathrm{g} / \mathrm{cm}^{2}\right)$ que los reales en $\mathrm{g} / \mathrm{cm}^{3}$. Esta situación ha tratado de ser corregida considerando separadamente la variable «tamaño óseo», sin resultados clínicamente útiles ${ }^{16}$. Sin embargo, al parecer, el error de apreciación de la DMO que 
esta limitación conlleva, no afecta la estimación del riesgo de fractura, por cuanto los huesos de menor tamaño, en los cuales la DMO estaría subestimada, pueden ser más frágiles ${ }^{16}$.

Se demuestra una correlación entre el área ósea y los parámetros antropométricos (peso, talla e índice de masa corporal), lo que es consistente por cuanto existe una relación entre el tamaño óseo y el tamaño corporal, aun cuando, como se ha mostrado, dicha correlación no parece ser estrictamente lineal, por cuanto el área ósea no corrige completamente los efectos de la variación del tamaño corporal. Esto puede deberse a que el área ósea es un parámetro bidimensional y las variaciones del tamaño óseo son tridimensionales, y además no es esperable necesariamente una correlación con ajuste lineal muy elevada entre el tamaño óseo y el tamaño corporal evaluado sólo en términos de peso y talla. Por otra parte, se observa una correlación positiva entre la edad y el área ósea, tanto en la columna vertebral como en cuello femoral, en ausencia de correlación entre la edad y las variables antropométricas. Esta relación podría reflejar un mayor tamaño óseo a mayor edad, derivado de cambios degenerativos (osteofitos, calcificación de ligamentos, engrosamiento perióstico). Sin embargo, también pueden influir cambios en las características de las partes blandas vecinas al hueso, por cuanto el área ósea se determina por una gradiente de densidades entre el hueso y las partes blandas. En un trabajo reciente realizado en 8.789 mujeres normales, entre 33 y 73 años, se demostró una relación inversa entre el área ósea y la edad ${ }^{3}$. Estos hallazgos, discordantes con los resultados de este trabajo, podrían explicarse por cuanto el rango etario evaluado en dicho estudio era más amplio, pudiendo esperarse en las personas de mayor edad una disminución del área ósea por cambios en la morfología vertebral y de los discos intervertebrales propios de la edad más avanzada. Dicha relación requeriría mayor investigación, porque existe la posibilidad de errores interpretativos del hallazgo de cambios en CMO o DMO en edades más avanzadas, que en parte podrían tener relación con cambios en el área ósea. Sería conveniente evaluar estas características mediante otros estudios adicionales de diseño transversal como éste, especialmente en evaluaciones longitudina- les, en que se estudie la posible variación del A y los factores que la influyen, en los mismos pacientes al avanzar la edad.

Estudios como el nuestro podrían presentar ciertas limitaciones relacionadas con el equipo utilizado, las características y tamaño de la población evaluada y el diseño propiamente tal. Entre las primeras, la más importante sería un defecto en la precisión o exactitud del equipo utilizado. En este trabajo es poco probable una causa de error de esta naturaleza por cuanto los controles de calidad y las determinaciones realizadas con un fantom internacional demuestran que dichos parámetros se encuentran plenamente dentro de los rangos esperados (Tabla 2).

La muestra evaluada podría no ser plenamente representativa de la población chilena normal, por cuanto fue obtenida en determinadas instituciones y mediante la convocación de voluntarios, ambos factores que pueden introducir sesgos en la selección de los individuos. Sin embargo, estimamos que para los objetivos de este trabajo, estas condiciones, así como el tamaño de la muestra, están dentro de lo razonable. Un aspecto limitante en nuestro estudio fue no disponer de una información detallada de la base de datos Hologic con los valores individuales y parámetros antropométricos, que permitirían una comparación muchísimo más adecuada entre las dos poblaciones y evitaría la necesidad de adoptar una determinada distribución de rangos etarios. Esta situación, sin embargo, no tiene solución, por cuanto dicha información no está disponible y sólo es posible acceder al promedio y desviación estándar en los rangos etarios definidos por Hologic.

En cuanto al tipo de estudio, se trata de una evaluación transversal, lo cual también lleva inherente ciertos errores al incluir individuos de distinta edad y no evaluar el efecto de la edad en un mismo sujeto, aunque tiene la ventaja de disminuir costos y tiempo de observación.

La densitometría ósea es una técnica de medición que se ha perfeccionado en diversos aspectos, constituyéndose actualmente en una metodología precisa y exacta en la estimación del grado de mineralización ósea. Sin embargo, para su correcta interpretación, en sus aplicaciones clínicas y epidemiológicas, deben considerarse los efectos que sobre ella ejercen un conjunto de 
variables entre las cuales se cuentan el tamaño y composición corporal del paciente y la base de datos normativa utilizada, debiéndose evaluar separadamente los 3 parámetros osteodensitométricos $\mathrm{CMO}$, área ósea y DMO, especialmente en los estudios de seguimiento, considerando que la DMO es un producto de la relación entre las otras dos variables.

Es conveniente que las bases de datos normativa utilizadas sean validadas localmente. Un proce-

\section{REFERENCIAS}

1. Blake GM, Fogelman I. Technical principles of dual energy X-ray absorptiometry. Sem Nucl Med 1997; 27: 210-28.

2. Prentice A, Parsons TJ, Coie TJ. Uncritical use of bone mineral density in absorptiometry may lead to sizerelated artifacts in the identification of bone mineral determinants. Am J Clin Nutr 1994; 60: 837-42.

3. Shipman AJ, Guy GWG, Smith I, Ostiere S, Greer W, SмITH R. Vertebral bone mineral density, content and area in 8789 normal women aged 33-73 years who have never had hormone replacement therapy. Osteoporos Int 1999; 9: 420-6.

4. SeBERT JL Mesure de la masse osseuse par absortiométrie biphotonique aux rayons X. Cahiers du Grio 1996; 34: 3-5.

5. Kolta S, Roux CH. Controle de qualité en densitométrie osseuse. J Radiol 1999; 80: 93-8.

6. Gajardo H, Barrera G. Control de calidad de la densitometría ósea: precisión, reproducibilidad y aplicación clínica. Rev Méd Chile 1998; 126: 56-62.

7. Ponce L, Larenas G, Ruedemann G. Alta prevalencia de osteoporosis en mujeres postmenopáusicas asintomáticas. Rev Méd Chile 2002; 130: 1365-72.

8. Rodríguez Portales JA. Hipovitaminosis D en mujeres postmenopáusicas con masa ósea baja en la región metropolitana. Rev Méd Chile 2001; 129: 849-52.

9. Ryan PJ. Overview of role of BMD measurements in managing osteoporosis. Sem Nucl Med 1997; 3: 197-209. dimiento simple consiste en evaluar la distribución de los Z score de una población local normal, como el efectuado en este trabajo, en que si la base de datos es adecuada debieran distribuirse en proporción equivalente los valores positivos y negativos. La discusión en torno a este tema, sin embargo, permanece vigente y algunos autores plantean el uso de las cifras absolutas de densidad ósea para establecer el riesgo de fractura, obviando la necesidad de valores de referencia ${ }^{15}$.

10. Milier PD, Bonnick SL, Rosen CJ. Consensus of an international panel on the clinical utility of bone mass measurements in the detection of low bone mass in the adult population. Calcif Tissue Int 1996; 58: 207-14.

11. VAN LOAN MD, Johnson HL, BarBIERI TF. Effect of weight loss on bone mineral density in obese women. Am J Clin Nutr 1998; 67: 734-8.

12. Biake GM, Fogelman I. Interpretation of bone densitometry studies. Sem Nucl Med 1997; 27: 248-60.

13. Ahmed AiH, Blake GM, Rymer JM, Fogelman I. Screening for osteopenia and osteoporosis. Do the accepted normal ranges lead to overdiagnosis? Osteoporos Int 1997; 7: 432-8.

14. FAuLKNer KG, Roberts LA, McClung MR. Discrepancies in normative data between Lunar and Hologic DXA systems. Osteoporos Int 1996; 6: 432-6.

15. Roig-Vilaseca D, Noшa JM, Roig-Escofet D. Suitability of the T-score for establishing bone mineral density categories. Osteoporos Int 2000; 11: 40810.

16. Compston JE. Bone density: BMC, BMD or corrected BM? Bone 1995; 16: 5-6.

Agradecimientos

$\mathrm{Al} \mathrm{Sr}$. Carlos Wolff por su asesoría estadística. Al Sr. Claudio Troncoso por su colaboración en la realización de los exámenes densitométricos y reclutamiento de personas normales. 